\title{
SCREEN CONFORMAL HALF-LIGHTLIKE SUBMANIFOLDS
}

\author{
K. L. DUGGAL and B. SAHIN
}

Received 18 March 2004

\begin{abstract}
We study some properties of a half-lightlike submanifold $M$, of a semi-Riemannian manifold, whose shape operator is conformal to the shape operator of its screen distribution. We show that any screen distribution $S(T M)$ of $M$ is integrable and the geometry of $M$ has a close relation with the nondegenerate geometry of a leaf of $S(T M)$. We prove some results on symmetric induced Ricci tensor and null sectional curvature of this class.
\end{abstract}

2000 Mathematics Subject Classification: 53C20, 53C50.

1. Introduction. The Riemannian geometry of submanifolds [3] is one of the most important topics of differential geometry. It is well known that semi-Riemannian submanifolds [9] have many similarities with their Riemannian case.

However, the lightlike submanifolds [6] are different since (contrary to the nondegenerate cases) their normal vector bundle intersects with the tangent bundle. Thus, one cannot use, in the usual way, the classical submanifold theory to define any induced object on a lightlike submanifold. To deal with this anomaly, the lightlike submanifolds were introduced and presented in a book by Duggal and Bejancu [6]. They introduced a nondegenerate screen distribution to construct a nonintersecting lightlike transversal vector bundle of the tangent bundle. Since then, a suitable choice of an integrable screen distribution has produced several new results on lightlike geometry (see, e.g, $[1,4,5,7]$ and many more references therein). Also, see [8] for a different approach to deal with lightlike (degenerate) submanifolds. However, unfortunately, there are only two papers [5, 7] (after the publication of Duggal-Bejancu's book [6]) on a subclass called half-lightlike submanifolds of codimension 2 (see Section 2) which provides a physical model of null 2-surfaces in 4-dimensional space-time manifolds (see Example 3.3).

The growing importance of geometry in mathematical physics and very limited information available on half-lightlike submanifolds are the motivation for the study on this topic. Since the shape operator plays a key role in the geometry of submanifolds $[3,6]$, the objective of this paper is to study those half-lightlike submanifolds, of a semi-Riemannian manifold, whose shape operator is conformal to the shape operator of their screen distribution.

In Section 2, we brief basic information needed for the rest of the paper. In Section 3, we define screen conformal half-lightlike submanifolds $M$ and prove that any screen distribution $S(T M)$ of $M$ is integrable (see Theorem 3.5). This result is important since it does not hold for an arbitrary lightlike submanifold. Then we prove (Theorem 3.6) that the geometry of $M$ is closely related with the nondegenerate geometry of a leaf $M^{\prime}$ of its integrable $S(T M)$. In particular, $M=L \oplus M^{\prime}$ is a product lightlike manifold if and 
only if there exists an induced Levi-Civita connection on $M$ (Theorem 3.9), where $L$ and $M^{\prime}$ are leaves of $\operatorname{Rad} T M$ and $S(T M)$, respectively. We also find conditions for the induced Ricci tensor to be symmetric and induced nonvanishing sectional curvature of $M$. Using Kupeli's [8] concept of an irrotational lightlike submanifold (see Definition 3.7) we show that the induced Ricci tensor of any screen conformal half-lightlike irrotational submanifold is symmetric. This result is desirable both for geometry and its physical interpretation. In Section 4, we show that some classical results of Riemannian submanifolds also hold for screen conformal totally umbilical half-lightlike submanifolds of a semi-Riemannian space form $\bar{M}(c)$.

2. Half-lightlike submanifolds. Let $(\bar{M}, \bar{g})$ be an $(m+2)$-dimensional $(m>1)$ semiRiemannian manifold of index $q \geq 1$ and $M$ a submanifold of codimension 2 of $\bar{M}$. In case $\bar{g}$ is degenerate on the tangent bundle $T M$ of $M$ we say that $M$ is a lightlike submanifold of $\bar{M}$ [6]. Throughout this paper we denote by $F(M)$ the algebra of smooth functions on $M$ and by $\Gamma(E)$ the $F(M)$-module of smooth sections of a vector bundle $E$ over $M$. We use the same notation for any other vector bundle. All manifolds are paracompact and smooth. For basic information on the geometry of submanifolds, we refer to [3]. Denote by $g$ the induced degenerate tensor field of $\bar{g}$ on $M$. Then, there exists locally (or globally) a vector field $\xi \in \Gamma(T M), \xi \neq 0$, such that $g(\xi, X)=0$ for any $X \in \Gamma(T M)$, and for each tangent space $T_{x} M$, we consider

$$
T_{x} M^{\perp}=\left\{u \in T_{x} \bar{M}: \bar{g}(u, v)=0, \forall v \in T_{x} M\right\}
$$

a degenerate 2-dimensional orthogonal (but not complementary) subspace of $T_{x} \bar{M}$. The radical subspace $\operatorname{Rad} T_{x} M=T_{x} M \cap T_{x} M^{\perp}$ depends on the point $x \in M$. The submanifold $M$ is said to be $r$-lightlike if the mapping

$$
\operatorname{Rad} T M: x \in M \longrightarrow \operatorname{Rad} T_{x} M
$$

defines a radical distribution on $M$ of rank $r>0$. $M$ is a half-lightlike submanifold of $\bar{M}$ [7] if $r=1$ and there exist $\xi, u \in T_{X} M^{\perp}$ such that

$$
\overline{\mathcal{g}}(\xi, v)=0, \quad \overline{\mathcal{g}}(u, u) \neq 0, \quad \forall v \in T_{X} M^{\perp} .
$$

The above relations imply that $\xi \in \operatorname{Rad} T_{x} M$. Thus,

$$
\overline{\mathfrak{g}}(\xi, X)=\overline{\mathfrak{g}}(\xi, v)=0, \quad \forall X \in \Gamma(T M), v \in \Gamma\left(T M^{\perp}\right) .
$$

Thus, $\operatorname{Rad} T M$ is locally (or globally) spanned by $\xi$. There exists a supplementary nondegenerate distribution to $\operatorname{Rad} T M$ in $T M$, called a screen distribution $S(T M)$ of $M$ [6] with the following orthogonal distribution:

$$
T M=\operatorname{Rad} T M \perp S(T M) .
$$

In this paper, we assume that $M$ is half-lightlike. Consider orthogonal complementary distribution $S\left(T M^{\perp}\right)$ to $S(T M)$ in $T \bar{M}$. Certainly $\xi$ and $u$ belong to $\Gamma\left(S\left(T M^{\perp}\right)\right)$. Choose 
$u$ as a unit vector field, with $\bar{g}(u, u)=\epsilon= \pm 1$. We brief the following results (for details see [7]). Consider a supplementary distribution $D$ to $\operatorname{Rad} T M$ in $S\left(T M^{\perp}\right)$ which is spanned by $u$. Hence we have the following orthogonal decomposition:

$$
S\left(T M^{\perp}\right)=D \perp D^{\perp}
$$

where $D^{\perp}$ is the orthogonal complementary distribution to $D$ in $S\left(T M^{\perp}\right)$. There exists a uniquely defined vector field $N \in \Gamma\left(D^{\perp}\right)$ satisfying

$$
\overline{\mathcal{g}}(N, \xi) \neq 0, \quad \overline{\mathcal{g}}(N, N)=\overline{\mathcal{g}}(N, u)=0 .
$$

Hence, $N$ is a lightlike vector field which is neither tangent to $M$ nor collinear with $u$ since $\bar{g}(u, \xi)=0$. Define a vector bundle $\operatorname{tr}(T M)$ of $M$ by

$$
\operatorname{tr}(T M)=D \perp \operatorname{ntr}(T M)
$$

where $\operatorname{ntr}(T M)$ is a 1-dimensional vector bundle generated by $N$. Therefore,

$$
\begin{aligned}
T \bar{M} & =S(T M) \perp(\operatorname{Rad} T M \oplus \operatorname{tr}(T M)) \\
& =S(T M) \perp D \perp(\operatorname{Rad} T M \oplus \operatorname{ntr}(T M)) .
\end{aligned}
$$

According to the decompositions (2.5) and (2.9) we choose the fields of frames $\left\{\xi, W_{1}\right.$, $\left.\ldots, W_{m-1}\right\}$ and $\left\{\xi, W_{1}, \ldots, W_{m-1}, u, N\right\}$ on $M$ and $\bar{M}$, respectively, where $\left\{W_{1}, \ldots, W_{m-1}\right\}$ is an orthonormal basis of $\Gamma(S(T M))$.

Although $S(T M)$ is not unique, it is canonically isomorphic to the factor vector bundle $T M / \operatorname{Rad} T M$ [8]. For the dependence of all the induced geometric objects of $M$ on $\left\{S(T M), S\left(T M^{\perp}\right)\right\}$, we refer to [6, Chapter 5]. In particular, the following result is important for this paper.

Proposition 2.1 [6, page 157]. The second fundamental forms of a lightlike submanifold $M$ do not depend on $S(T M), S\left(T M^{\perp}\right)$, and $\operatorname{ntr}(T M)$.

Let $P$ be the projection of $T M$ on $S(T M)$. It follows from (2.9) that

$$
X=P X+\eta(X) \xi, \quad \eta(X)=\bar{g}(X, N), \quad \forall X \in \Gamma(T M) .
$$

According to (2.5) and (2.9) we put

$$
\begin{gathered}
\bar{\nabla}_{X} Y=\nabla_{X} Y+h(X, Y), \\
\bar{\nabla}_{X} N=-A_{N} X+\nabla_{X} N, \\
\bar{\nabla}_{X} u=-A_{u} X+\nabla_{X} u, \quad \forall X, Y \in \Gamma(T M),
\end{gathered}
$$

where $\nabla_{X} Y, A_{N} X$, and $A_{u} X$ belong to $\Gamma(T M)$, while $h(X, Y), \nabla_{X} N$, and $\nabla_{X} u$ belong to $\Gamma(\operatorname{tr}(T M)), \bar{\nabla}$ is the Levi-Civita connection on $\bar{M}$ and $\nabla$ is a torsion-free linear connection on $M$. Define symmetric $F(M)$-bilinear forms $D_{1}$ and $D_{2}$ and 1 -forms $\rho_{1}, \rho_{2}, \varepsilon_{1}$, and $\varepsilon_{2}$ 
by

$$
\begin{aligned}
D_{1}(X, Y)=\bar{g}(h(X, Y), \xi), & D_{2}(X, Y)=\epsilon \bar{g}(h(X, Y), u), \\
\rho_{1}(X)=\bar{g}\left(\nabla_{X} N, \xi\right), & \rho_{2}(X)=\epsilon \overline{\mathcal{g}}\left(\nabla_{X} N, u\right), \\
\varepsilon_{1}(X)=\bar{g}\left(\nabla_{X} u, \xi\right), & \varepsilon_{2}(X)=\epsilon \bar{g}\left(\nabla_{X} u, u\right),
\end{aligned}
$$

for any $X, Y \in \Gamma(T M)$. It follows that

$$
\begin{aligned}
h(X, Y) & =D_{1}(X, Y) N+D_{2}(X, Y) u, \\
\nabla_{X} N & =\rho_{1}(X) N+\rho_{2}(X) u, \\
\nabla_{X} u & =\varepsilon_{1}(X) N+\varepsilon_{2}(X) u .
\end{aligned}
$$

Hence (2.11) become

$$
\begin{aligned}
& \bar{\nabla}_{X} Y=\nabla_{X} Y+D_{1}(X, Y) N+D_{2}(X, Y) u, \\
& \bar{\nabla}_{X} N=-A_{N} X+\rho_{1}(X) N+\rho_{2}(X) u, \\
& \bar{\nabla}_{X} u=-A_{u} X+\varepsilon_{1}(X) N+\varepsilon_{2}(X) u,
\end{aligned}
$$

for any $X, Y \in \Gamma(T M)$. We call $h, D_{1}$, and $D_{2}$ the second fundamental form, the lightlike second fundamental form, and the screen second fundamental form of $M$ with respect to $\operatorname{tr}(T M)$, respectively. Both $A_{N}$ and $A_{u}$ are linear operators on $\Gamma(T M)$ and $A_{N}$ is called the shape operator of $M$. Since $u$ is a unit vector field, (2.16) implies $\varepsilon_{2}(X)=0$. In a similar way, since $\xi$ and $N$ are lightlike vector fields, from (2.14)-(2.16) we obtain

$$
\begin{gathered}
D_{1}(X, \xi)=0, \quad \overline{\mathcal{g}}\left(A_{N} X, N\right)=0, \\
\overline{\mathcal{g}}\left(A_{u} X, Y\right)=\epsilon D_{2}(X, Y)+\varepsilon_{1}(X) \eta(Y) .
\end{gathered}
$$

Next, by using (2.10), (2.14)-(2.16), and (2.18), we obtain

$$
\begin{gathered}
\rho_{1}(X)=-\eta\left(\nabla_{X} \xi\right), \quad \rho_{2}(X)=\epsilon \eta\left(A_{u} X\right), \quad \varepsilon_{1}(X)=-\epsilon D_{2}(X, \xi), \\
\left(\nabla_{X} g\right)(Y, Z)=D_{1}(X, Y) \eta(Z)+D_{1}(X, Z) \eta(Y)
\end{gathered}
$$

for any $X, Y, Z \in \Gamma(T M)$. Thus, in general, the induced connection $\nabla$ is not a metric (Levi-Civita) connection. From (2.14) it follows that $D_{1}$ and $D_{2}$ are symmetric bilinear forms on $\Gamma(T M)$. From (2.5) we obtain

$$
\begin{gathered}
\nabla_{X} P Y=\nabla_{X}^{*} P Y+h^{*}(X, P Y), \\
\nabla_{X} \xi_{1}=-A_{\xi}^{*} X+\nabla_{X}^{\perp} \xi, \quad \forall X, Y \in \Gamma(T M),
\end{gathered}
$$

where $\nabla_{X}^{*} P Y$ and $A_{\xi}^{*}$ belong to $\Gamma(S(T M))$, while $h^{*}(X, P Y)$ and $\nabla_{X}^{\perp} \xi_{1}$ belong to $\Gamma(\operatorname{Rad}$ $T M)$. It follows that $\nabla^{*}$ and $\nabla^{\perp}$ are linear connections on the screen and radical distributions, respectively, $A_{\xi}^{*}$ is the shape operator of $S(T M)$, and $\nabla^{*}$ is a metric connection on $S(T M)$. Define

$$
E(X, P Y)=\bar{g}\left(h^{*}(X, P Y), N\right), \quad u_{1}(X)=\bar{g}\left(\nabla_{X}^{\perp} \xi, N\right),
$$


for all $X, Y \in \Gamma(T M)$. It follows that

$$
h^{*}(X, P Y)=E(X, P Y) \xi, \quad \nabla_{X}^{\perp} \xi_{1}=u_{1}(X) \xi, \quad \forall X, Y \in \Gamma(T M) .
$$

Hence the two equations of (2.21) become

$$
\begin{gathered}
\nabla_{X} P Y=\nabla_{X}^{*} P Y+E(X, P Y) \xi, \\
\nabla_{X} \xi=-A_{\xi}^{*} X+u_{1}(X) \xi,
\end{gathered}
$$

where $h^{*}$ and $E$ are the second fundamental form and the local second fundamental form of $S(T M)$ with respect to $\operatorname{Rad} T M$, and $A_{\xi}^{*}$ is the shape operator of the screen distribution. Equations (2.14)-(2.16) on one side and (2.24)-(2.25) on the other side are related by

$$
\begin{gathered}
E(X, P Y)=g\left(A_{N} X, P Y\right), \\
D_{1}(X, P Y)=g\left(A_{\xi}^{*} X, P Y\right), \\
u_{1}(X)=-\rho_{1}(X), \quad \forall X, Y \in \Gamma(T M) .
\end{gathered}
$$

From (2.17) and (2.27) we derive

$$
A_{\xi}^{*} \xi=0
$$

THEOREM 2.2 [6]. Let $M$ be a half-lightlike submanifold of $\bar{M}$. Then the following assertions are equivalent:

(1) the induced connection $\nabla$ on $M$ is a metric connection;

(2) $D_{1}$ vanishes identically on $M$;

(3) $A_{\xi}^{*}$ vanishes identically on $M$;

(4) $\xi$ is a Killing vector field;

(5) $T M^{\perp}$ is a parallel distribution with respect to $\nabla$.

Thus, contrary to the case of a lightlike hypersurface (see [6, page 88]), a half-lightlike submanifold satisfying one of the conditions of Theorem 2.2 is not totally geodesic unless $D_{2}$ vanishes on $M$. In particular, the existence of an induced connection on $M$, with nonvanishing second fundamental form $h$, is one of the important results of halflightlike submanifolds.

3. Conformal screen shape operator. It is well known that the second fundamental form and its shape operator of a nondegenerate submanifold are related by means of the metric tensor field. Contrary to this we see from Section 2 that in the case of half-lightlike submanifolds $M$ the second fundamental forms of $M$ and their screen distribution $S(T M)$ are related to their respective shape operators $A_{N}$ and $A_{\xi}^{*}$. As the shape operator is an information tool in studying the geometry of submanifolds, in this paper we consider a class of half-lightlike submanifolds with conformal screen shape operator defined as follows.

DEFINITION 3.1. A half-lightlike submanifold $M$ of a semi-Riemannian manifold is screen locally (resp., globally) conformal if on any coordinate neighborhood $u$ (resp., $u=M$ ) there exists a nonzero smooth function $\varphi$ such that for any null vector field 
$\xi \in \Gamma\left(T M^{\perp}\right)$ the relation

$$
A_{N} X=\varphi A_{\xi}^{*} X, \quad \forall X \in \Gamma\left(\left.T M\right|_{\iota}\right),
$$

holds between the shape operators $A_{N}$ and $A_{\xi}^{*}$ of $M$ and $S(T M)$, respectively.

In the case of half-lightlike submanifolds, since $A_{N} X$ and $A_{\xi}^{*} X$ belong to screen distribution for any $X \in \Gamma(T M)$, this definition is well defined.

EXAMPLE 3.2. Consider in $R_{2}^{5}$ a submanifold $M$ given by the equations

$$
x_{4}=\left(x_{1}^{2}+x_{2}^{2}\right)^{1 / 2}, \quad x_{3}=\left(1-x_{5}^{2}\right)^{1 / 2}, \quad x_{5}, x_{1}, x_{2}>0 .
$$

Then we have

$$
\begin{gathered}
T M=\operatorname{Span}\left\{\xi=x_{1} \frac{\partial}{\partial x_{1}}+x_{2} \frac{\partial}{\partial x_{2}}+x_{4} \frac{\partial}{\partial x_{4}},\right. \\
\left.U=x_{4} \frac{\partial}{\partial x_{1}}+x_{1} \frac{\partial}{\partial x_{4}}, V=-x_{5} \frac{\partial}{\partial x_{3}}+x_{3} \frac{\partial}{\partial x_{5}}\right\}, \\
T M^{\perp}=\operatorname{Span}\left\{\xi, u=x_{3} \frac{\partial}{\partial x_{3}}+x_{5} \frac{\partial}{\partial x_{5}}\right\} .
\end{gathered}
$$

Thus $\operatorname{Rad} T M=\operatorname{Span}\{\xi\}$ is a distribution on $M$ and $S\left(T M^{\perp}\right)=\operatorname{Span}\{u\}$. Hence $M$ is a half-lightlike submanifold of $R_{2}^{5}$, with $S(T M)=\operatorname{Span}\{U, V\}$. Also, the lightlike transversal bundle $\operatorname{ntr}(T M)$ is spanned by

$$
N=\frac{1}{2 x_{2}^{2}}\left\{x_{1} \frac{\partial}{\partial x_{1}}-x_{2} \frac{\partial}{\partial x_{2}}+x_{4} \frac{\partial}{\partial x_{4}}\right\}
$$

By direct calculations, we obtain

$$
\begin{aligned}
& \bar{\nabla}_{U} \xi=U, \quad \bar{\nabla}_{V} \xi=0, \quad \bar{\nabla}_{\xi} \xi=\xi, \\
& \bar{\nabla}_{U} N=\frac{1}{2 x_{2}^{2}} U, \quad \bar{\nabla}_{V} N=0, \quad \bar{\nabla}_{\xi} N=-N \text {. }
\end{aligned}
$$

Then, from (2.15) and (2.25) we obtain

$$
\begin{gathered}
A_{\xi}^{*} U=-U, \quad A_{\xi}^{*} V=0, \\
A_{N} U=-\frac{1}{2 x_{2}^{2}} U, \quad \rho_{1}(U)=0, \quad \rho_{2}(U)=0, \\
A_{N} V=0, \quad \rho_{1}(V)=0, \quad \rho_{2}(V)=0, \\
A_{N} \xi=0, \quad \rho_{1}(\xi)=-1, \quad \rho_{2}(\xi)=0 .
\end{gathered}
$$

Hence we derive $A_{N} X=\left(1 / 2 x_{2}^{2}\right) A_{\xi}^{*} X$, for all $X \in \Gamma(T M)$. Thus $M$ is a screen conformal lightlike submanifold with $\varphi=1 / 2 x_{2}^{2}$.

EXAMPLE 3.3. Following [5, Section 3] one can show that an $n$-dimensional halflightlike submanifold of a Minkowski space-time $\mathbb{R}_{1}^{n+2}$ is screen lightlike with a constant 
conformal factor. In particular, a null 2-surface in 4-dimensional space-time manifold is a physical example of such half-lightlike submanifolds. Indeed, consider a 4dimensional space-time $(\bar{M}, \bar{g})$, with Lorentzian metric $\bar{g}$, of signature $(-,+,+,+)$, which admits a smooth 2-parameter group $G$, generated by two spacelike Killing vector fields $U$ and $V$. Suppose $\bar{M}$ also admits a non-Killing null vector field $\xi$. Then, $U$ and $V$ will span a lightlike surface $M$ defined by $U_{\left[a V_{b}\right]} U^{a} V^{b}=0, a, b \in\{1,2,3,4\}$. At any $x$ of $T_{x} M$ we have a unique null vector tangent to $T_{x} M$ given by

$$
\xi=U-\Omega V, \quad \Omega=\left(V^{a} V_{a}\right)^{-1} U^{b} V_{b}, \quad \bar{g}(\xi, U)=0=\bar{g}(\xi, V) .
$$

It is easy to see that $M$ is a half-lightlike surface of $\bar{M}$ such that $\operatorname{Rad} T M=\operatorname{Span}\{\xi\}$. Since $\xi$ is non-Killing, it follows from Theorem 2.2 that $D_{1} \neq 0$ and, therefore, $M$ neither admits a metric connection nor is totally geodesic.

Proposition 3.4. Let $M$ be a half-lightlike submanifold of a semi-Riemannian manifold $\bar{M}$. Then, $M$ is screen conformal if and only if

$$
E(X, P Y)=\varphi D_{1}(X, P Y), \quad \forall X, Y \in \Gamma(T M) .
$$

Proof. Suppose $M$ is a screen conformal half-lightlike submanifold. Then, from (2.26), (2.27), and (3.1), we get

$$
E(X, P Y)=g\left(A_{N} X, P Y\right)=\varphi g\left(A_{\xi}^{*} X, P Y\right)=\varphi D_{1}(X, P Y)
$$

for all $X, Y \in \Gamma(T M)$. Conversely, if $E(X, P Y)=\varphi D_{1}(X, P Y)$, for all $X, Y \in \Gamma(T M)$, then (2.26) and (2.27) imply $g\left(A_{N} X, P Y\right)=g\left(\varphi A_{\xi}^{*} X, P Y\right)$. Thus, we get $A_{N} X=\varphi A_{\xi}^{*} X$, which completes the proof.

Let $M$ be screen conformal. Then, from (2.24) and (3.8) we get

$$
\nabla_{X} P Y=\nabla_{X}^{*} P Y+\varphi D_{1}(X, P Y) \xi, \quad \forall X, Y \in \Gamma(T M)
$$

THEOREM 3.5. Any screen distribution of a screen conformal half-lightlike submanifold $M$ of a semi-Riemannian manifold $\bar{M}$ is integrable.

Proof. Using (2.14) and (3.10) and for any $X, Y, Z \in \Gamma(T M)$, we obtain

$$
\begin{aligned}
\bar{g}([X, Y], N) & =\bar{g}\left(\nabla_{X} Y, N\right)-\bar{g}\left(\nabla_{Y} X, N\right) \\
& =\varphi D_{1}(X, Y) \bar{g}(\xi, N)-\varphi D_{1}(Y, X) \bar{g}(\xi, N) \\
& =\varphi\left\{D_{1}(X, Y)-D_{1}(Y, X)\right\} .
\end{aligned}
$$

Since $D_{1}$ is symmetric, we get $\bar{g}([X, Y], N)=0$. Hence $S(T M)$ is integrable.

THEOREM 3.6. Let $M$ be a screen conformal half-lightlike submanifold of a semiRiemannian manifold $\bar{M}$, with a leaf $M^{\prime}$ of $S(T M)$. Then

(1) $M$ is totally geodesic,

(2) $M$ is totally umbilical,

(3) $M$ is minimal,

if and only if $M^{\prime}$ is so immersed as a submanifold of $\bar{M}$ and $\varepsilon_{1}$ vanishes on $M$. 
Proof. Using (3.8) we obtain

$$
\bar{\nabla}_{X} Y=\nabla_{X}^{*} Y+\varphi D_{1}(X, Y) \xi+D_{1}(X, Y) N+D_{2}(X, Y) u
$$

for any $X, Y \in \Gamma\left(T M^{\prime}\right)$. Then we have

$$
\bar{\nabla}_{X} Y=\nabla_{X}^{\prime} Y+h^{\prime}(X, Y)
$$

where $h^{\prime}$ and $\nabla^{\prime}$ are second fundamental form and the Levi-Civita connection of $M^{\prime}$ in $\bar{M}$. Thus, from (3.12) and (3.13) we obtain

$$
h^{\prime}(X, Y)=(\varphi \xi+N) D_{1}(X, Y)+D_{2}(X, Y) u
$$

for any $X, Y \in \Gamma\left(T M^{\prime}\right)$. On the other hand, from (2.18) we have

$$
\epsilon D_{2}(\xi, P Z)=g\left(A_{u} \xi, P Z\right), \quad \epsilon D_{2}(P Z, \xi)=-\varepsilon_{1}(P Z) .
$$

Since $D_{2}$ is symmetric, we obtain $-\varepsilon_{1}(P Z)=g\left(A_{u} \xi, P Z\right)$. Similarly we get $\epsilon D_{2}(\xi, \xi)=$ $\varepsilon_{1}(\xi)$. Consequently, we obtain

$$
D_{2}(\xi, P Z)=D_{2}(P Z, \xi)=D_{2}(\xi, \xi)=0 \Longleftrightarrow \varepsilon_{1}(Z)=0 \quad \forall Z \in \Gamma(T M)
$$

Thus the proof follows from (3.14) and (3.16).

DEFINITION 3.7. A lightlike submanifold $M$ is said to be irrotational if $\bar{\nabla}_{X} \xi \in \Gamma(T M)$ for any $X \in \Gamma(T M)$, where $\xi \in \Gamma(\operatorname{Rad} T M)$ [8].

For a half-lightlike $M$, since $D_{1}(X, \xi)=0$, the above definition is equivalent to $D_{2}(X$, $\xi)=0=\varepsilon_{1}(X)$, for all $X \in \Gamma(T M)$. Using this in (3.16) we state the following corollary.

COROLLARY 3.8. Let $M$ be an irrotational screen conformal half-lightlike submanifold of a semi-Riemannian manifold $\bar{M}$. Then

(1) $M$ is totally geodesic,

(2) $M$ is totally umbilical,

(3) $M$ is minimal,

if and only if a leaf $M^{\prime}$ of any $S(T M)$ is so immersed as a submanifold of $\bar{M}$.

THEOREM 3.9. Let $M$ be a screen conformal half-lightlike submanifold of a semiRiemannian manifold $\bar{M}$. Then, the following assertions are equivalent:

(1) any leaf of $S(T M)$ is totally geodesic in $M$;

(2) $M$ is a lightlike product manifold of $M^{\prime}$ and $L$, where $M^{\prime}$, a leaf of $S(T M)$, is a nondegenerate manifold and $L$ is a one-dimensional lightlike manifold;

(3) $D_{1}$ vanishes identically on $M$;

(4) the induced connection $\nabla$ on $M$ is a metric connection.

Proof. From (2.14) we have $g\left(\nabla_{\xi} \xi, X\right)=\bar{g}\left(\bar{\nabla}_{\xi} \xi, X\right)$ for $X \in \Gamma(S(T M))$ and $\xi \in$ $\Gamma(\operatorname{Rad}(T M))$. Now $\bar{\nabla}$, a metric connection, implies $g\left(\nabla_{\xi} \xi, X\right)=-\bar{g}(\xi, \bar{\nabla} \xi X)$. Now, using 
(2.14) and (2.17), we obtain $g(\nabla \xi \xi, X)=-D_{1}(\xi, X) \bar{g}(\xi, N)$. Thus, from (2.17), we get

$$
g\left(\nabla_{\xi} \xi, X\right)=0
$$

Similarly, from (2.15) we derive $\bar{g}\left(\nabla_{X} Y, N\right)=\mathcal{g}\left(A_{N} X, Y\right)$ for all $X, Y \in \Gamma(S(T M))$ and $N \in \Gamma(\operatorname{ltr}(T M))$. Then, from (3.1), we obtain $\bar{g}\left(\nabla_{X} Y, N\right)=\varphi g\left(A_{\xi}^{*} X, Y\right)$. Thus, from (2.27), we get

$$
\overline{\mathfrak{g}}\left(\nabla_{X} Y, N\right)=\varphi D_{1}(X, Y) .
$$

Now, from (3.17) and (3.18), the equivalence of (1) and (2) follows. If $M$ is a lightlike product, then any leaf of $S(T M)$ is parallel. Thus, from (3.18) $D_{1}=0$ since $D_{1}(X, \xi)=0$. Conversely, if $D_{1}=0$, then from (3.18) a leaf of $S(T M)$ is parallel and considering (3.17) we obtain (2). Thus (2) is equivalent to (3). Finally, the equivalence of (3) and (4) comes from Theorem 2.2.

Denote by $\bar{R}$ and $R$ the curvature tensors of $\bar{\nabla}$ and $\nabla$, respectively. Then, using (2.14)(2.16) and (2.18)-(2.25), we obtain

$$
\begin{aligned}
\bar{R}(X, Y) Z= & R(X, Y) Z+D_{1}(X, Z) A_{N} Y-D_{1}(Y, Z) A_{N} X \\
+ & D_{2}(X, Z) A_{u} Y-D_{2}(Y, Z) A_{u} X \\
+ & \left\{\left(\nabla_{X} D_{1}\right)(Y, Z)-\left(\nabla_{Y} D_{1}\right)(X, Z)\right. \\
& +\rho_{1}(X) D_{1}(Y, Z)-\rho_{1}(Y) D_{1}(X, Z) \\
& \left.+\varepsilon(X) D_{2}(Y, Z)-\varepsilon(Y) D_{2}(X, Z)\right\} N \\
+ & \left\{\left(\nabla_{X} D_{2}\right)(Y, Z)-\left(\nabla_{Y} D_{2}\right)(X, Z)\right. \\
& \left.+\rho_{2}(X) D_{1}(Y, Z)-\rho_{2}(Y) D_{1}(X, Z)\right\} u .
\end{aligned}
$$

Let $M$ be screen conformal. Consider the Riemannian curvature of type $(0,4)$ of $\bar{\nabla}$, and by using (3.19) and the definition of curvature tensors, we derive the following structure equations:

$$
\begin{aligned}
\bar{g}(\bar{R}(X, Y) Z, P W)= & g(R(X, Y) Z, P W) \\
& +\varphi\left\{D_{1}(X, Z) D_{1}(Y, P W)-D_{1}(Y, Z) D_{1}(X, P W)\right\} \\
& +\epsilon\left\{D_{2}(X, Z) D_{2}(Y, P W)-D_{2}(Y, Z) D_{2}(X, P W)\right\}, \\
\bar{g}(\bar{R}(X, Y) P Z, N)= & \bar{g}(R(X, Y) P Z, N) \\
& +\epsilon\left\{\rho_{2}(Y) D_{2}(X, P Z)-\rho_{2}(X) D_{2}(Y, P Z)\right\} \\
= & g\left(\nabla_{X}\left(A_{N} Y\right)-\nabla_{Y}\left(A_{N} X\right)-A_{N}[X, Y], P Z\right) \\
& +\varphi\left\{\rho_{1}(Y) D_{1}(X, P Z)-\rho_{1}(X) D_{1}(Y, P Z)\right\} \\
& +\epsilon\left\{\rho_{2}(Y) D_{2}(X, P Z)-\rho_{2}(X) D_{2}(Y, P Z)\right\}, \\
\bar{g}(\bar{R}(X, Y) \xi, P Z)= & g(R(X, Y) \xi, P Z)+\epsilon D_{2}(X, \xi) D_{2}(Y, P Z) \\
& -\epsilon D_{2}(Y, \xi) D_{2}(X, P Z) .
\end{aligned}
$$


Let $R^{*}$ be the curvature tensor of $\nabla^{*}$. Using (2.24) and (3.8) we obtain

$$
\begin{aligned}
R(X, Y) P Z= & R^{*}(X, Y) P Z-\varphi\left\{D_{1}(Y, P Z) A_{\xi}^{*} X-D_{1}(X, P Z) A_{\xi}^{*} Y\right\} \\
& +\varphi\left\{\left(\nabla_{X} D_{1}\right)(Y, P Z)-\left(\nabla_{Y} D_{1}\right)(X, P Z)\right\} \xi \\
& +D_{1}(Y, P Z)\left\{X(\varphi)-\varphi \rho_{1}(X)\right\} \xi \\
& -D_{1}(X, P Z)\left\{Y(\varphi)-\varphi \rho_{1}(Y)\right\} \xi
\end{aligned}
$$

THEOREM 3.10. Let $M$ be a screen conformal half-lightlike submanifold of a semiRiemannian space form $\bar{M}(c)$. Then, the induced Ricci tensor of $M$ is symmetric if and only if

$$
\left(D_{2} \wedge \rho_{2}\right)(\xi, X, Y)=D_{2}(X, Y) \rho_{2}(\xi)
$$

Proof. The Ricci tensor of a half-lightlike submanifold is given by

$$
\operatorname{Ric}(X, Y)=\sum_{i=1}^{m-1} \varepsilon \mathcal{g}\left(R\left(X, e_{i}\right) Y, e_{i}\right)+\bar{g}(R(X, \xi) Y, N), \quad \forall X, Y \in \Gamma(T M)
$$

For a space form $\bar{M}(c)$, from (3.8), (3.20), and (3.21), we have

$$
\begin{aligned}
\operatorname{Ric}(X, Y)= & (1-m) c g(X, Y) \\
+ & +\sum_{i=1}^{m-1}\left\{\left(-D_{1}(X, Y) D_{1}\left(e_{i}, e_{i}\right)+D_{1}\left(e_{i}, Y\right) D_{1}\left(X, e_{i}\right)\right) \varphi\right. \\
& \left.-\varepsilon\left(D_{2}(X, Y) D_{2}\left(e_{i}, e_{i}\right)+D_{2}\left(e_{i}, Y\right) D_{2}\left(X, e_{i}\right)\right)\right\} \\
& -\varepsilon D_{2}(X, Y) \rho_{2}(\xi)+\varepsilon D_{2}(\xi, Y) \rho_{2}(X) .
\end{aligned}
$$

Thus we get

$$
\begin{aligned}
\operatorname{Ric}(X, Y)-\operatorname{Ric}(Y, X) & =\varepsilon\left\{D_{2}(\xi, Y) \rho_{2}(X)-D_{2}(\xi, X) \rho_{2}(Y)\right\} \\
& =\left(D_{2} \wedge \rho_{2}\right)(\xi, X, Y)-D_{2}(X, Y) \rho_{2}(\xi),
\end{aligned}
$$

which proves the theorem.

The following result holds from Definition 3.7 and (3.27).

COROLLARY 3.11. The Ricci tensor of any irrotational screen conformal half-lightlike submanifold $M$ of $\bar{M}(c)$ is symmetric.

Let $p \in M$ and let $\xi$ be a null vector of $T_{p} M$. A plane $H$ of $T_{p} M$ is called a null plane directed by $\xi$ if it contains $\xi, \bar{g}(\xi, W)=0$ for any $W \in H$ and there exits $W_{0} \in H$ such that $\bar{g}\left(W_{0}, W_{0}\right) \neq 0$. Then the null sectional curvature of $H$ with respect to $\xi$ and $\bar{\nabla}$ is defined by [2, page 431]

$$
K_{\xi}(H)=\frac{R_{p}(W, \xi, \xi, W)}{g_{p}(W, W)}
$$


THEOREM 3.12. Let $M$ be a screen conformal half-lightlike submanifold of a space form $\bar{M}(c)$. Then, the null sectional curvature of $M$ is given by

$$
K_{\xi}(H)=\epsilon\left\{D_{2}(\xi, \xi) D_{2}(X, X)-D_{2}(X, \xi) D_{2}(\xi, X)\right\},
$$

for $X \in \Gamma(S(T M))$ and $\xi \in \Gamma(\operatorname{Rad} T M)$.

Proof. From (3.20) we have

$$
\begin{aligned}
K_{\xi}(H)= & \varphi\left\{D_{1}(X, \xi) D_{1}(\xi, X)-D_{1}(\xi, \xi) D_{1}(X, X)\right\} \\
& +\epsilon\left\{D_{2}(\xi, \xi) D_{2}(X, X)-D_{2}(X, \xi) D_{2}(\xi, X)\right\} .
\end{aligned}
$$

Using (2.17) we obtain (3.29), which proves the theorem.

Moreover, using (2.19) in (3.29) and Definition 3.7, we have the following corollary.

COROLLARY 3.13. The null sectional curvature of a screen conformal half-lightlike submanifold $M$ of $\bar{M}(c)$ vanishes identically if and only if

$$
\left(D_{2} \wedge \varepsilon_{1}\right)(X, \xi, X)=-\epsilon \varepsilon_{1}^{2}(X), \quad \forall X \in \Gamma(S(T M)), \xi \in \Gamma(\operatorname{Rad} T M) .
$$

Consequently, the null sectional curvature of any irrotational conformal half-lightlike submanifold of $\bar{M}(c)$ vanishes identically.

THEOREM 3.14. Let $M$ be a screen conformal half-lightlike submanifold of $\bar{M}(c)$ with $D_{2}=0$. Then, $M$ is flat if and only if $M^{\prime}$ is flat and $c=0$.

Proof. Suppose $M$ is flat. For $\bar{M}(c)$, from (3.21) we derive

$$
\begin{aligned}
g(R(X, Y) P Z, N)= & -\epsilon D_{2}(X, P Z) \rho_{2}(Y)+\epsilon D_{2}(Y, P Z) \rho_{2}(X) \\
& +c\{g(Y, P Z) \eta(X)-g(X, P Z) \eta(Y)\}=0 .
\end{aligned}
$$

Since $D_{2}=0$ and $M$ is flat, we obtain

$$
c\{g(Y, P Z) \eta(X)-g(X, P Z) \eta(Y)\}=0 .
$$

Thus, for $X=\xi$ and $Y=P Z$, we derive $c g(P Z, P Z)=0$, hence $c=0$. On the other hand, from (3.20) we have

$$
\begin{aligned}
g(R(X, Y) P Z, P W)= & c\{g(Y, P Z) g(X, P W)-g(X, P Z) g(Y, P W)\} \\
& -\varphi\left\{D_{1}(Y, P W) D_{1}(X, P Z)-D_{1}(Y, P Z) D_{1}(X, P W)\right\} \\
& -\epsilon D_{2}(X, P Z) D_{2}(Y, P W) \\
& +\epsilon D_{2}(Y, P Z) D_{2}(X, P W) .
\end{aligned}
$$

Using (3.34) in (3.23) we get

$$
\begin{aligned}
2 g(R(X, Y) P Z, P W)= & g\left(R^{*}(X, Y) P Z, P W\right)+\epsilon D_{2}(X, P W) D_{2}(Y, P Z) \\
& -D_{2}(X, P Z) D_{2}(Y, P W) \\
& +c\{g(Y, P Z) g(X, P W)-g(X, P Z) g(Y, P W)\} .
\end{aligned}
$$


Thus, from (3.35) we have $R^{*}=0$ due to $c=0$ and $D_{2}=0$. Now suppose that $M^{\prime}$ is flat and $c=0$. Using (3.32) and (3.35) we obtain

$$
g(R(X, Y) P Z, P W)=0, \quad g(R(X, Y) P Z, N)=0 .
$$

On the other hand, since $\bar{M}$ is a space form and $D_{1}(X, \xi)=0$, we have

$$
\overline{\mathcal{g}}(R(X, Y) \xi, N)=0, \quad \forall X \in \Gamma(T M) .
$$

Moreover, since $D_{2}=0$, from (3.22) we get

$$
\mathcal{g}(R(X, Y) \xi, P Z)=0
$$

Thus, (3.36)-(3.38) imply $R=0$, which proves the theorem.

EXAMPLE 3.15. Consider the screen conformal half-lightlike submanifold $M$ of $R_{2}^{5}$ given in Example 3.2, and by direct calculations, we obtain

$$
\begin{gathered}
\bar{\nabla}_{U} V=\bar{\nabla}_{V} U=\bar{\nabla}_{\xi} V=\bar{\nabla}_{V} \xi=0, \quad \bar{\nabla}_{U} \xi=U, \\
\bar{\nabla}_{U} U=\frac{1}{2} \xi+x_{2}^{2} N, \quad \bar{\nabla}_{V} V=-u, \quad \bar{\nabla}_{\xi} \xi=\xi, \\
\bar{\nabla}_{U} u=0, \quad \bar{\nabla}_{V} u=V, \quad \bar{\nabla}_{\xi} u=0 .
\end{gathered}
$$

Thus, from (2.14)-(2.16), (2.25), and (2.26) we derive

$$
\begin{gathered}
\nabla_{U} U=\frac{1}{2} \xi, \quad E(U, U)=\frac{1}{2}, \quad A_{u} U=0, \quad A_{u} V=-V, \quad A_{u} \xi=0 \\
D_{1}(U, U)=x_{2}^{2}, \quad D_{1}(V, V)=0, \quad D_{2}(U, U)=0, \quad D_{2}(V, V)=-1 \\
D_{2}(X, \xi)=0, \quad \epsilon_{1}(X)=0, \quad \forall X \in \Gamma(T M) .
\end{gathered}
$$

Hence $M$ is irrotational with a symmetric Ricci tensor (Theorem 3.10) and vanishing null sectional curvature (Corollary 3.13). $D_{1} \neq 0$ implies that $\nabla$ is not a metric connection and $M$ is not totally geodesic. Also $M^{\prime}$ is not totally geodesic in $\bar{M}$ (Theorem 3.6). Moreover, $S(T M)$ is not parallel in $M$ due to $E(U, U) \neq 0$. Thus $M$ is not a lightlike product (Theorem 3.9).

4. Totally umbilical submanifolds. Following the definition of totally umbilical Riemannian submanifolds [3], we say that a lightlike submanifold $(M, g)$ of a semiRiemannian manifold $(\bar{M}, \bar{g})$ is said to be totally umbilical in $\bar{M}$ if there is a smooth transversal vector field $\mathscr{H} \in \Gamma(\operatorname{tr}(T M))$ on $M$, called the transversal curvature vector field of $M$, such that, for all $X, Y \in \Gamma(T M)$,

$$
h(X, Y)=\mathscr{H} \bar{g}(X, Y) .
$$

The above definition does not depend on the screen distribution and the transversal bundle of $M$. Let $M$ be a half-lightlike submanifold. Using (2.14) and (4.1), we conclude 
that $M$ is totally umbilical if and only if there exist smooth functions $H_{1} \in \Gamma(\operatorname{ltr}(T M))$ and $H_{2} \in \Gamma\left(S\left(T M^{\perp}\right)\right)$ such that

$$
\begin{aligned}
& D_{1}(X, Y)=g(X, Y) H_{1}, \\
& D_{2}(X, Y)=g(X, Y) H_{2},
\end{aligned}
$$

for any $X, Y \in \Gamma(T M)$. We have the following existence theorem.

THEOREM 4.1. Let $M$ be a half-lightlike submanifold of a semi-Riemannian manifold $\bar{M}$. Suppose $S(T M)$ is integrable and any leaf $M^{\prime}$ of $S(T M)$ is totally umbilical immersed in $\bar{M}$ as a codimension 3 nondegenerate submanifold with $\alpha \beta>0$. Then $M$ is screen locally conformal if and only if $E(\xi, P X)=0$ for $\xi \in \Gamma(\operatorname{Rad} T M)$ and $X \in \Gamma(T M)$, where $\alpha$ and $\beta$ are components of mean curvature vector field of the leaf, in the direction of $\xi$ and $N$.

Proof. Let $M^{\prime}$ be a leaf of $S(T M)$. Then we have

$$
\bar{\nabla}_{X} Y=\nabla_{X}^{*} Y+E(X, Y) \xi+D_{1}(X, Y) N+D_{2}(X, Y) u
$$

for any $X, Y \in \Gamma\left(T M^{\prime}\right)$. The mean curvature vector field $H^{*}$ is $H^{*}=\alpha \xi+\beta N+\gamma u$. Since $M^{\prime}$ is totally umbilical in $\bar{M}$, we get

$$
E(X, Y) \xi+D_{1}(X, Y) N+D_{2}(X, Y) u=g(X, Y)\{\alpha \xi+\beta N+\gamma u\} .
$$

Thus we have

$$
\begin{gathered}
E(X, Y)=\alpha g(X, Y), \\
D_{1}(X, Y)=\beta g(X, Y), \quad D_{2}(X, Y)=\gamma g(X, Y) .
\end{gathered}
$$

Equations (4.6) imply $E(X, Y)=(\alpha / \beta) D_{1}(X, Y)$. Hence, $E(X, Y)=(\alpha / \beta) D_{1}(X, Y)$ for all $X, Y \in \Gamma\left(T M^{\prime}\right)$. Since $A_{\xi}^{*} \xi=0$ and $E(\xi, Y)=0$, we obtain $A_{N} X=\varphi A_{\xi}^{*} X$ for $X \in$ $\Gamma(T M)$. Conversely, if $M$ is screen conformal, then it can be seen that $E(\xi, X)=0$, which completes the proof.

DEFINITION 4.2. A leaf $M^{\prime}$ of an integrable screen distribution $S(T M)$ of a lightlike submanifold $M$ of a semi-Riemannian manifold $\bar{M}$ is totally umbilical in $M$ if and only if on any coordinate neighborhood $U \subset M$ there is a smooth function $K$ such that

$$
E(X, P Y)=K g(X, P Y) .
$$

For a screen conformal $M,(3.8)$ implies that $M^{\prime}$ is totally umbilical if

$$
D_{1}(X, P Y)=\frac{K}{\varphi} g(X, P Y), \quad \forall X \in \Gamma(T M) .
$$

THEOREM 4.3. Let $M$ be a screen conformal half-lightlike submanifold of $\bar{M}$. Then $M$ is totally umbilical if and only if

$$
P\left(A_{u} X\right)=H_{2} P X, \quad \varepsilon_{1}(X)=0, \quad X \in \Gamma(T M),
$$

and a leaf $M^{\prime}$ of any $S(T M)$ is totally umbilical in $M$. 
Proof. From (2.18) we obtain that $D_{2}(X, Y)=g(X, Y) H_{2}$ if and only if $P\left(A_{u} X\right)=$ $H_{2} P X$ and $\varepsilon_{1}(X)=0$, for all $X \in \Gamma(T M)$ (also proved in [7]). Suppose $D_{1}(X, Y)=$ $g(X, Y) H_{1}$. Then, $M$ is screen conformal and (3.8) implies $E(X, Y)=\varphi H_{1} g(X, Y)$. Hence $M^{\prime}$ is totally umbilical with $K=\varphi H_{1}$. Conversely, if $M^{\prime}$ is totally umbilical, then using (3.8), (4.7), and (2.17) we obtain $D_{1}(X, Y)=H_{1} g(X, Y)$, where $H_{1}=K / \varphi$, which completes proof.

THEOREM 4.4. Let $M$ be a screen conformal totally umbilical half-lightlike submanifold of a semi-Riemannian manifold $\bar{M}$. Then

(1) $M^{\prime}$ is totally umbilical in $\bar{M}$;

(2) $M$ is totally geodesic if and only if $M^{\prime}$ is totally geodesic in $\bar{M}$.

Proof. For totally umbilical $M, D_{2}(X, \xi)=0$. Thus, from (3.14), (4.2), and (4.3), we obtain $h^{\prime}(X, Y)=g(X, Y)\left(H_{1} \varphi \xi+H_{1} N+H_{2} u\right)$, for all $X, Y \in \Gamma\left(T M^{\prime}\right)$, which completes the proof.

THEOREM 4.5. Let $M(c)$ be a screen conformal half-lightlike submanifold of constant curvature c of $\bar{M}$. Suppose $M^{\prime}$ is totally umbilical in $M(c)$. Then $c=0$, that is, the submanifold is a semi-Euclidean space if and only if the mean curvature vector $K$ is a solution of the partial differential equation

$$
\xi(K)-K \rho_{1}(\xi)-K^{2} \varphi^{-1}=0 .
$$

Proof. From (3.23) we have

$$
\begin{aligned}
\overline{\mathcal{g}}(R(X, Y) P Z, N)= & \varphi\left\{\left(\nabla_{X} D_{1}\right)(Y, P Z)-\left(\nabla_{Y} D_{1}\right)(X, P Z)\right\} \\
& +D_{1}(Y, P Z)\left\{X(\varphi)-\varphi \rho_{1}(X)\right\} \\
& -D_{1}(X, P Z)\left\{Y(\varphi)-\varphi \rho_{1}(Y)\right\}
\end{aligned}
$$

for $X, Y, Z \in \Gamma(T M)$. On the other hand, using (2.20) and (4.8) we obtain

$$
\begin{aligned}
\left(\nabla_{X} D_{1}\right)(Y, P Z)= & \left(X(K) \varphi^{-1}+X\left(\varphi^{-1}\right) K\right) g(Y, P Z) \\
& +K^{2}(\varphi)^{-2} g(X, P Z) \eta(Y) .
\end{aligned}
$$

Thus, by direct calculations, using (4.8), (4.11), and (4.12), we derive

$$
\begin{aligned}
\overline{\mathcal{g}}(R(X, Y) P Z, N)= & \left\{X(K)-K^{2} \varphi^{-1} \eta(X)-K \rho_{1}(X)\right\} \mathcal{g}(Y, P Z) \\
& +\left\{K^{2} \varphi^{-1} \eta(Y)-Y(K)+K \rho_{1}(Y)\right\} \mathcal{g}(X, P Z) .
\end{aligned}
$$

Since $M$ is of constant curvature, for $X=\xi$, we obtain

$$
\operatorname{cg}(Y, P Z)=\left\{\xi(K)-K^{2} \varphi^{-1}-K \rho_{1}(\xi)\right\} g(Y, P Z),
$$

which proves the assertion of the theorem. 
Let $M$ be a totally umbilical half-lightlike submanifold in $\bar{M}(c)$. Then, by direct calculations, using (2.14), (2.15), (2.16), and (4.2) and taking the tangential parts, we obtain

$$
\begin{aligned}
R(X, Y) Z= & c\{g(Y, Z) X-g(X, Z) Y\}-g(X, Z) H_{1} A_{N} Y \\
& +g(Y, Z) H_{1} A_{N} X+H_{2}\left\{g(Y, Z) A_{u} X-g(X, Z) A_{u} Y\right\}
\end{aligned}
$$

Now, from (2.19), (4.15), (4.3), (2.27), (3.1), and (4.8) we obtain

$$
\begin{aligned}
g(R(X, Y) Z, W)= & c\{g(Y, Z) g(X, W)-g(X, Z) g(Y, W)\} \\
& -g(X, Z) \varphi g(Y, W)\left(H_{1}\right)^{2}+g(Y, Z) \varphi g(X, W)\left(H_{1}\right)^{2} \\
& -g(X, Z) H_{2} \in D_{2}(Y, W)+g(Y, Z) H_{2} \in D_{2}(X, W),
\end{aligned}
$$

for all $X, Y, Z \in \Gamma(T M)$ and $W \in \Gamma(S(T M))$. Thus we obtain

$$
\mathfrak{g}(R(X, Y) Z, W)=[g(Y, Z) g(X, W)-g(X, Z) g(Y, W)] \times\left[c+\varphi\left(H_{1}\right)^{2}+\epsilon\left(H_{2}\right)^{2}\right]
$$

On the other hand, from (3.23) we obtain

$$
\mathcal{g}(R(X, Y) Z, W)=R^{*}((X, Y) Z, W)-\varphi g(Y, Z) H_{1} g\left(A_{\xi}^{*} X, W\right)+\varphi g(X, Z) H_{1} g\left(A_{\xi}^{*} Y, W\right)
$$

for all $X, Y \in \Gamma(T M)$ and $Z, W \in \Gamma(S(T M))$. Here, using (2.27) and (3.1) we get

$$
g(R(X, Y) Z, W)=R^{*}((X, Y) Z, W)-\varphi\left(H_{1}\right)^{2}[g(Y, Z) g(X, W)-g(X, Z) g(Y, W)]
$$

Thus, from (4.17) and (4.19), we obtain

$$
g\left(R^{*}(X, Y) Z, W\right)=\{g(Y, Z) g(X, Z)-g(X, Z) g(Y, W)\} \times\left\{c+2 \varphi\left(H_{1}\right)^{2}+\epsilon\left(H_{2}\right)^{2}\right\}
$$

for all $X, Y \in \Gamma(T M)$ and $Z, W \in \Gamma(S(T M))$. As a result of (4.20) we have the following result.

THEOREM 4.6. Let $M$ be a screen conformal totally umbilical half-lightlike submanifold of a semi-Riemannian space form $\bar{M}(c)$. If $\operatorname{dim}\left(M^{\prime}\right)>2$, then $M^{\prime}$ is a semi-Riemannian space form if and only if $\varphi=$ constant.

From the proofs of Theorems 3.10 and 3.12, the following results hold.

(a) Let $M$ be a screen conformal totally umbilical half-lightlike submanifold of $\bar{M}(c)$. Then, the Ricci tensor of $M$ is symmetric.

(b) Let $M$ be a screen conformal totally umbilical half-lightlike submanifold of $\bar{M}(c)$. Then, the null sectional curvature of $M$ vanishes identically. 
EXAMPLE 4.7. Consider in $R_{1}^{4}$ a surface $M$ given by the equations

$$
x_{1}=x_{3}, \quad x_{2}=\left(1-x_{4}^{2}\right)^{1 / 2} .
$$

Then we have

$$
\begin{aligned}
T M & =\operatorname{Span}\left\{\xi=\frac{\partial}{\partial x_{1}}+\frac{\partial}{\partial x_{3}}, u=-x_{4} \frac{\partial}{\partial x_{2}}+x_{2} \frac{\partial}{\partial x_{4}}\right\}, \\
T M^{\perp} & =\operatorname{Span}\left\{\xi=\frac{\partial}{\partial x_{1}}+\frac{\partial}{\partial x_{3}}, v=x_{2} \frac{\partial}{\partial x_{2}}+x_{4} \frac{\partial}{\partial x_{4}}\right\} .
\end{aligned}
$$

Thus, $M$ is a half-lightlike submanifold of $R_{1}^{4}$ with $\operatorname{Rad} T M=\operatorname{Span}\{\xi\}$ and

$$
\begin{aligned}
S(T M) & =\operatorname{Span}\{u\}, \quad S\left(T M^{\perp}\right)=\operatorname{Span}\{v\}, \\
\operatorname{ntr}(T M) & =\operatorname{Span}\left\{N=\frac{1}{2}\left(-\frac{\partial}{\partial x_{1}}+\frac{\partial}{\partial x_{3}}\right)\right\} .
\end{aligned}
$$

Hence, we obtain $A_{\xi}^{*} u=A_{N} u=0$. Thus $M$ is a trivial screen conformal half-lightlike submanifold. On the other hand, by direct calculations, we derive

$$
D_{1}=0, \quad D_{2}(\xi, X)=0, \quad \forall X \in \Gamma(T M), \quad D_{2}(u, u)=-g(u, u) .
$$

Thus $M$ is a screen conformal totally umbilical half-lightlike submanifold. Moreover, $D_{1}=0$ implies that $\nabla$ is a metric connection.

CONCLUdING REMARKS. Recently, Duggal [4, 5] studied the following problem: find a class of lightlike submanifolds whose geometry is essentially the same as that of their chosen screen distribution $S(T M)$.

The above problem is motivated due to the fact that the lightlike geometry directly depends on a suitable choice of screen distribution, which plays an important role. For example, it has been shown in [6, page 133] that the geometry of a Monge lightlike hypersurface of $R_{1}^{4}$ essentially reduces to the geometry of a leaf of its canonical screen distribution (see also [1, 4] for some more results on the above-stated problem). In [5], a technique was used to show that some aspects of the geometry of a half-lightlike submanifold of a Minkowski space-time are the same as those of a leaf of its chosen integrable screen distribution. In the present paper, we have shown that any screen distribution of a screen conformal half-light submanifold $M$ of a semi-Riemannian manifold is integrable (Theorem 3.5) and the geometry of $M$ has a close relation with the nondegenerate geometry of a leaf of their screen distribution $S(T M)$ (Theorems 3.6 and 4.3). Thus, we have made further progress in solving the above-stated problem.

Finally, as per Proposition 2.1, the second fundamental forms of a lightlike submanifold $M$ do not depend on the vector bundles $S(T M), S\left(T M^{\perp}\right)$, and $\operatorname{ntr}(T M)$. Thus, the results of this paper are stable with respect to any change in the above vector bundles.

ACKNOWLEDGMENTS. The research of the first author was supported by the Natural Sciences and Engineering Research Council of Canada. The second author is a Postdoctoral Fellow at the University of Windsor, Canada (TUBITAK-NATO(B1)). 


\section{REFERENCES}

[1] C. Atindogbe and K. L. Duggal, Conformal screen on lightlike hypersurfaces, Int. J. Pure Appl. Math. 11 (2004), no. 4, 421-442.

[2] J. K. Beem and P. E. Ehrlich, Global Lorentzian Geometry, Monographs and Textbooks in Pure and Applied Math., vol. 67, Marcel Dekker, New York, 1981.

[3] B.-Y. Chen, Geometry of Submanifolds, Marcel Dekker, New York, 1973.

[4] K. L. Duggal, Constant scalar curvature and warped product globally null manifolds, J. Geom. Phys. 43 (2002), no. 4, 327-340.

[5] _ Riemannian geometry of half lightlike manifolds, Math. J. Toyama Univ. 25 (2002), 165-179.

[6] K. L. Duggal and A. Bejancu, Lightlike Submanifolds of Semi-Riemannian Manifolds and Applications, Mathematics and Its Applications, vol. 364, Kluwer Academic Publishers, Dordrecht, 1996.

[7] K. L. Duggal and D. H. Jin, Half lightlike submanifolds of codimension 2, Math. J. Toyama Univ. 22 (1999), 121-161.

[8] D. N. Kupeli, Singular Semi-Riemannian Geometry, Mathematics and Its Applications, vol. 366, Kluwer Academic Publishers, Dordrecht, 1996.

[9] B. O'Neill, Semi-Riemannian Geometry. With Applications to Relativity, Pure and Applied Mathematics, vol. 103, Academic Press, New York, 1983.

K. L. Duggal: Department of Mathematics and Statistics, University of Windsor, Windsor, Ontario, Canada N9B 3P4

E-mail address: yq8@uwindsor.ca

B. Sahin: Department of Mathematics, Inonu University, 44100 Malatya, Turkey

E-mail address: bsahin@inonu.edu.tr 


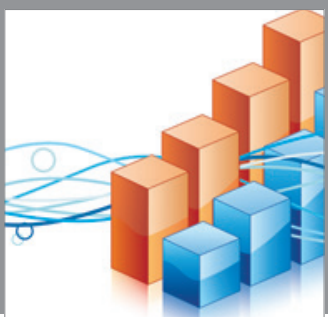

Advances in

Operations Research

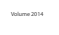

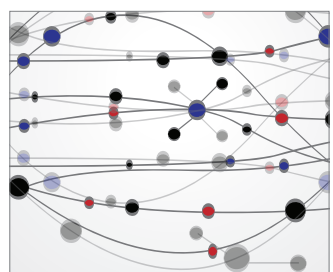

\section{The Scientific} World Journal
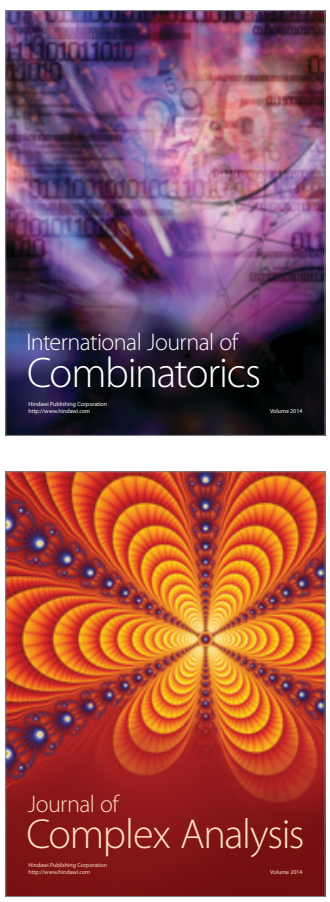

International Journal of

Mathematics and

Mathematical

Sciences
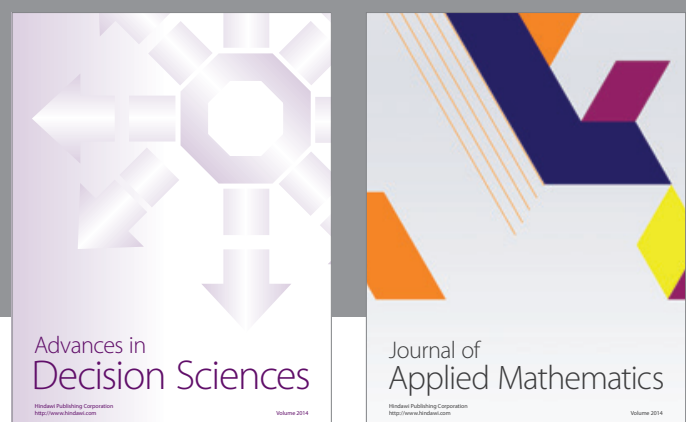

Journal of

Applied Mathematics
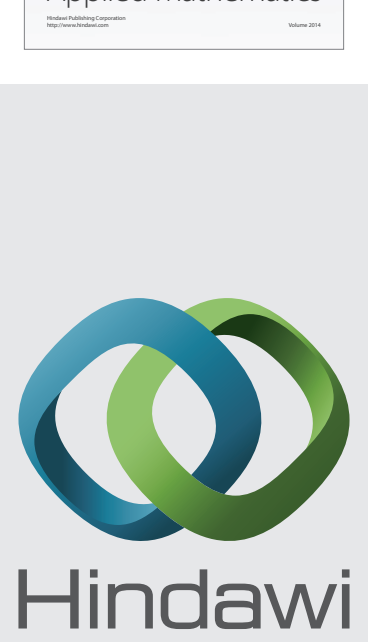

Submit your manuscripts at http://www.hindawi.com
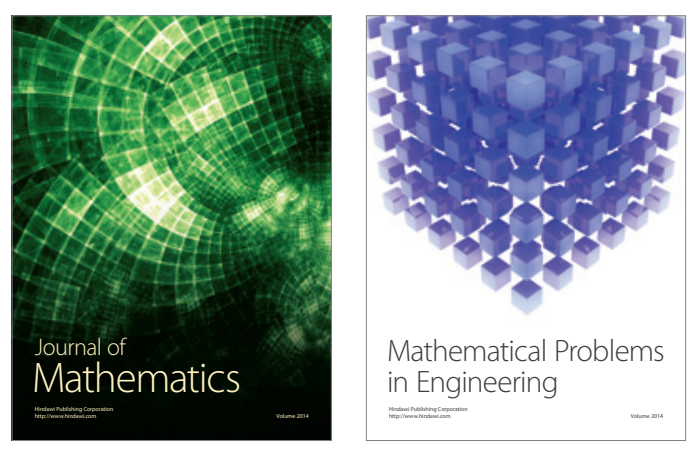

Mathematical Problems in Engineering
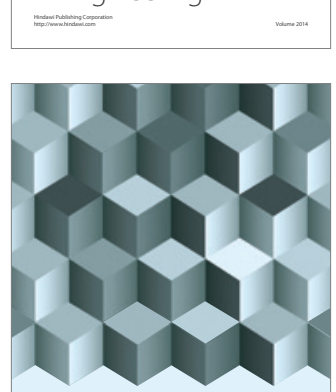

Journal of

Function Spaces
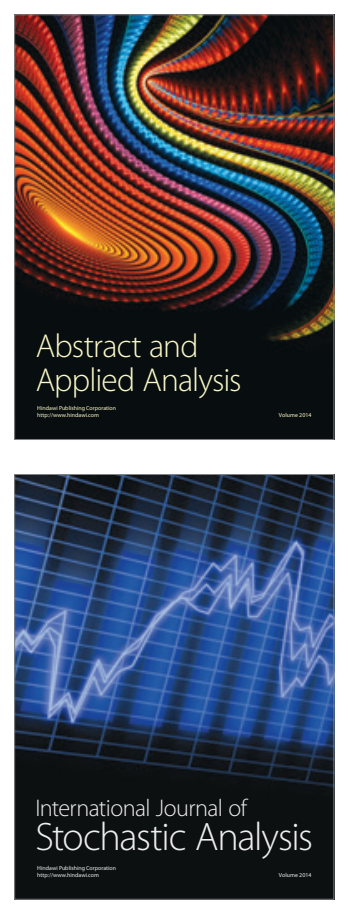

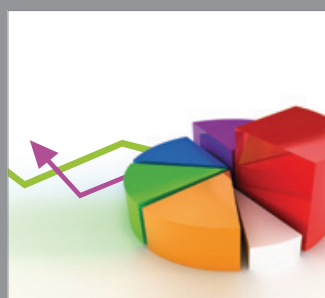

ournal of

Probability and Statistics

Promensencen
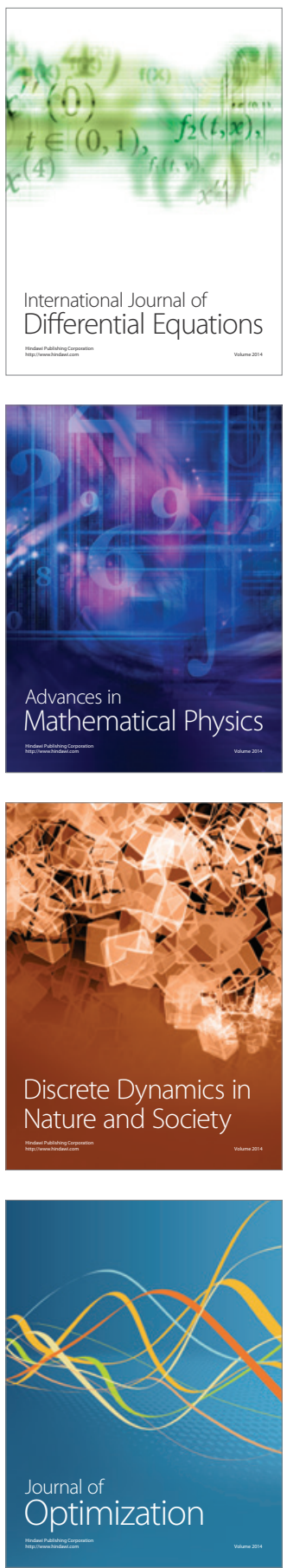